\title{
Performance of InGaN Light-Emitting Diodes Fabricated on Patterned Sapphire Substrates with Modified Top-Tip Cone Shapes
}

\author{
Hsu-Hung Hsueh, ${ }^{1}$ Sin-Liang Ou, ${ }^{2}$ Chiao-Yang Cheng, ${ }^{3}$ \\ Dong-Sing Wuu, ${ }^{2}$ and Ray-Hua Horng ${ }^{1,4}$ \\ ${ }^{1}$ Graduate Institute of Precision Engineering, National Chung Hsing University, 250 Kuo Kuang Road, Taichung 40227, Taiwan \\ ${ }^{2}$ Department of Materials Science and Engineering, National Chung Hsing University, Taichung 40227, Taiwan \\ ${ }^{3}$ Wafer Works Optronics Corporation, Taoyuan 32542, Taiwan \\ ${ }^{4}$ Advanced Optoelectronic Technology Center, National Cheng Kung University, Tainan 70101, Taiwan
}

Correspondence should be addressed to Ray-Hua Horng; huahorng@nchu.edu.tw

Received 14 February 2014; Revised 5 June 2014; Accepted 6 June 2014; Published 19 June 2014

Academic Editor: Hao-Chung Kuo

Copyright (C) 2014 Hsu-Hung Hsueh et al. This is an open access article distributed under the Creative Commons Attribution License, which permits unrestricted use, distribution, and reproduction in any medium, provided the original work is properly cited.

InGaN light-emitting diodes (LEDs) were fabricated on cone-shaped patterned sapphire substrates (PSSs) by using low-pressure metalorganic chemical vapor deposition. To enhance the crystal quality of the GaN epilayer and the optoelectronic performance of the LED device, the top-tip cone shapes of the PSSs were further modified using wet etching. Through the wet etching treatment, some dry-etched induced damage on the substrate surface formed in the PSS fabrication process can be removed to achieve a high epilayer quality. In comparison to the LEDs prepared on the conventional sapphire substrate (CSS) and cone-shaped PSS without wet etching, the LED grown on the cone-shaped PSS by performing wet etching for 3 min exhibited 55\% and $10 \%$ improvements in the light output power (at $350 \mathrm{~mA}$ ), respectively. This implies that the modification of cone-shaped PSSs possesses high potential for LED applications.

\section{Introduction}

In recent years, InGaN light-emitting diodes (LEDs) have been used in a wide range of optoelectronic applications such as traffic signals, automobiles, full-color displays, solidstate lighting, and backlights of liquid-crystal displays [13]. To apply for these applications, an LED device with high luminescence efficiency is required. Nevertheless, depositing a GaN epilayer on lattice-mismatched substrates consisting of sapphire and silicon carbide has resulted in a high threading dislocation (TD) density with a range from $10^{9}$ to $10^{11} \mathrm{~cm}^{-2}$ $[4,5]$. The high TD density created in the GaN epilayer causes a considerable deterioration of LED performance, including electron mobility, device lifetime, and the quantum efficiency of radiative recombination. Consequently, decreasing the TD density may be a key process in achieving high-efficiency LEDs.

To reduce the TD density of the GaN epilayer to a range of $10^{6}-10^{7} \mathrm{~cm}^{-2}$, several methods including epitaxial lateral overgrowth (ELOG), pendeoepitaxy, and facet-controlled ELOG have been developed. Moreover, because of its singlegrowth process with no interruption, the patterned sapphire substrate (PSS) technique is another promising method for achieving a GaN epilayer with high crystal quality. However, as the GaN epilayer is grown on PSS, a long period is required for merging the GaN epilayers grown on etched and nonetched sapphire and subsequently obtaining a smooth film surface. Based on previous reports [6-8], InGaN/GaN epilayers with high crystal quality can be achieved by using metalorganic chemical vapor deposition (MOCVD) on a 
cone-shaped PSS. At the first step of GaN growth on a coneshaped PSS, the epitaxial film is merely deposited on the flat basal of the sapphire substrate. Furthermore, the GaN growth on the cone regions has no preferential orientation. This indicates that the growth time of a GaN epilayer with a smooth surface on a cone-shaped PSS is less than that required for using the conventional PSS.

In this study, cone-shaped PSSs were fabricated using dry and wet etching processes and then employed for growing InGaN LED epitaxial structures. In addition, to improve the epilayer quality and LED performance, the wet etching process was applied to modify the top-tip shape of the PSSs by changing the etching time. The epilayer quality, light extraction characteristic, and optoelectronic performance were investigated in detail for these InGaN LEDs fabricated on PSSs with modified top-tip cone shapes.

\section{Experimental Procedure}

For the fabrication of PSSs with modified top-tip cone shapes, the dry and wet etching processes were employed in sequence, which are described as follows. First, a thick photoresist was deposited on the (001) sapphire through spincoating, and then a thermal photoresist reflow process was used to create the cone-shaped pattern array. The photoresist array was used as the mask layer to transfer the pattern on the sapphire by applying an inductively coupled plasma reactive ion etching (ICP-RIE) system using reactive $\mathrm{Cl}_{2}$ gas. The diameter, interval, and height of each cone-shaped pattern were set to $2.4,0.5$, and $1.5 \mu \mathrm{m}$, respectively. After performing ICP-RIE, the cone-shaped PSSs were further chemically etched using a mixture of $\mathrm{H}_{2} \mathrm{SO}_{4}: \mathrm{H}_{3} \mathrm{PO}_{4}(3: 1)$ solution at $250^{\circ} \mathrm{C}$ for $3,5,7$, and $10 \mathrm{~min}$ to form the various top-tip shapes. To clean the substrate surface, these PSSs were soaked in the $\mathrm{H}_{2} \mathrm{SO}_{4}: \mathrm{H}_{2} \mathrm{O}_{2}(3: 1)$ solution at $120^{\circ} \mathrm{C}$ for $10 \mathrm{~min}$ and in deionized water at room temperature for $10 \mathrm{~min}$ in sequence.

For growing the LED epitaxial structure, the epilayers were on these PSSs and on a conventional sapphire substrate (CSS) employed as the contrast sample by using low-pressure MOCVD. The LED epitaxial structure included a $3 \mu \mathrm{m}$ thick layer of undoped $\mathrm{GaN}$ (u-GaN), a $2 \mu \mathrm{m}$ thick layer of n-type $\mathrm{GaN}: \mathrm{Si}, 6$ periods of InGaN/GaN multiple quantum wells, a $100 \mathrm{~nm}$ thick p-type AlGaN layer, and a $0.2 \mu \mathrm{m}$ thick p-type GaN:Mg layer. To fabricate the LED device, a $24 \times 45$ mil. $^{2}$ mesa pattern was defined and prepared using standard photolithography and dry etching processes. Subsequently, the ITO film used as the transparent conducting layer was deposited on the p-type GaN layer. Finally, the $\mathrm{Cr} / \mathrm{Au}$ metal was prepared as both the $\mathrm{n}$ - and p-pad electrodes.

The epilayer quality of the flat $\mathrm{u}-\mathrm{GaN}$ was measured using X-ray diffraction (XRD) combined with the analyses of the (002) and (102) planes. The surface morphology and pattern feature of these PSSs were observed using scanning electron microscopy (SEM). The microstructure and electron diffraction pattern of the GaN epilayers grown on PSSs were investigated using transmission electron microscopy (TEM). The typical current-voltage $(I-V)$ characteristic of the fabricated InGaN LED device was analyzed using a semiconductor parameter analyzer (Keithley, 2400 sourcemeter), and the measurement of light output power was conducted using a calibrated integrating sphere.

\section{Results and Discussion}

Figure 1 shows the surface morphologies of the fabricated PSSs with various cone shapes. Figure 1(a) presents the crosssectional SEM image of the cone-shaped PSS that was not subjected to the wet etching process. We determined that the actual diameter and height of each cone-shaped pattern were 2.421 and $1.488 \mu \mathrm{m}$, respectively. The plane-view SEM images of the cone-shaped PSSs after further wet etching treatments administered using etching times of 3, 5, 7, and $10 \mathrm{~min}$ are shown in Figures 1(b), 1(c), 1(d), and 1(e), respectively. After conducting the wet etching process for $3 \mathrm{~min}$, the top-tip surface of the cone pattern was smooth. By increasing the etching time to 5-10 $\mathrm{min}$, we observed that the top-tip shape became increasingly angular. Additionally, the diameter of each cone pattern was enlarged and the interval between these patterns was decreased as the etching time was increased. Furthermore, inclined planes were formed on the sidewalls of the patterns when the etching time was increased, particularly for the samples that underwent 7-10 min of wet etching (Figures $1(\mathrm{~d})$ and $1(\mathrm{e})$ ).

The crystal quality of the GaN epilayer was evaluated using the full-width at half-maximum (FWHM) values for the XRD rocking curves. The XRD rocking curves derived on the GaN (002) and GaN (102) planes of all the samples are displayed in Figures 2(a) and 2(b), respectively. When the GaN epilayer was grown on the CSS, the FWHM values at the (002) and (102) planes were determined to be 412 and 593 arcsec, respectively. After growing the GaN epilayers on the PSSs by performing wet etching for $0,3,5,7$, and $10 \mathrm{~min}$, the FWHM values at the (002) plane were analyzed and determined to be 318,272, 279, 293, and $305 \mathrm{arcsec}$, respectively. The FWHM values at the (102) plane of the GaN epilayers deposited on these PSSs were 362, 285, 292, 322 , and 336 arcsec, respectively. The crystal quality of the GaN epilayer can clearly be improved by adopting the design for a cone-shaped PSS. In addition, we observed that the crystal quality of the GaN epilayer was further enhanced by performing the wet etching treatment on the PSS. According to our knowledge, some dry-etched induced damage on the substrate surface occurred when the ICP-RIE process was conducted to fabricate the PSS. Consequently, by performing the wet etching treatment on the PSS, both the modification of the top tip of the cone-shaped PSS and the removal of the dry-etched induced damage can be achieved. This is the reason that the crystal quality of the GaN epilayer was further improved by using the cone-shaped PSS combined with wet etching treatment. In addition, as mentioned previously, the inclined planes gradually formed on the sidewalls of the PSS as the wet etching time increased (Figure 1). These inclined planes clearly formed on the PSSs when wet etching was performed for 7-10 $\mathrm{min}$. The result indicates that the epilayer can most likely be deposited on both the bottom ( $c$-plane) 


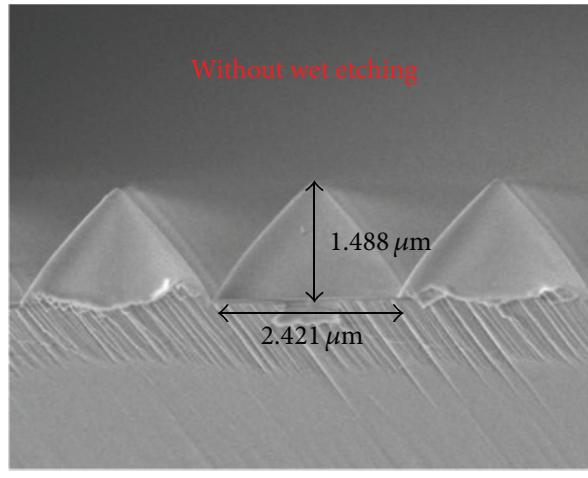

(a)

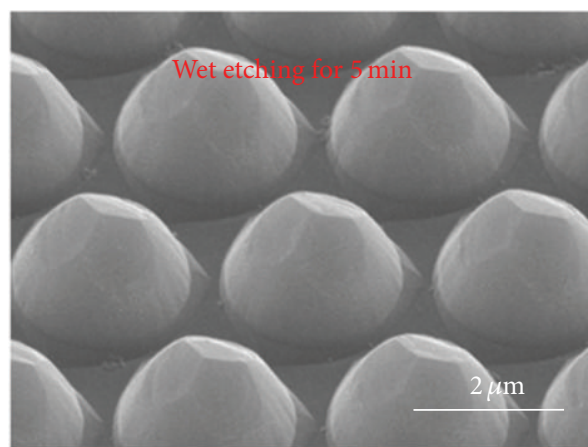

(c)

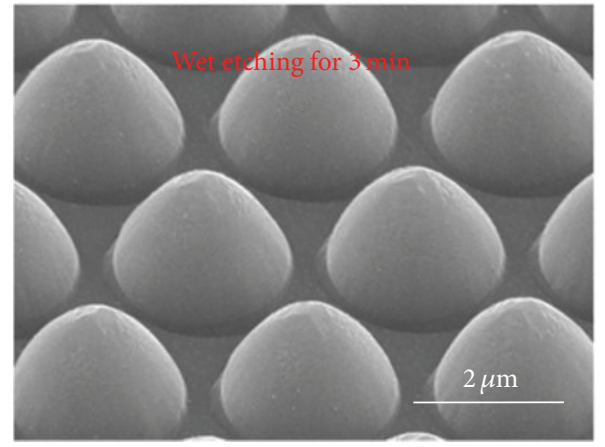

(b)

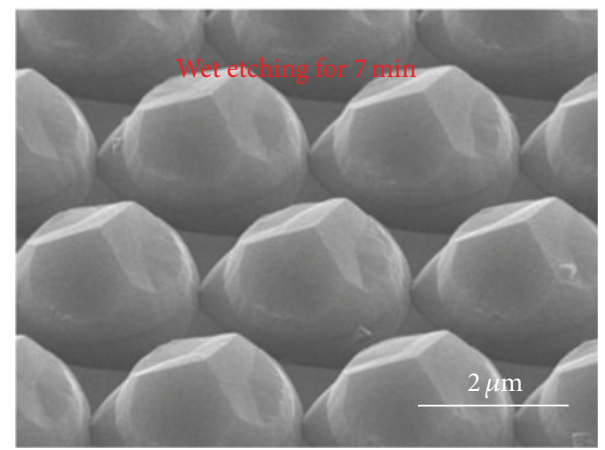

(d)

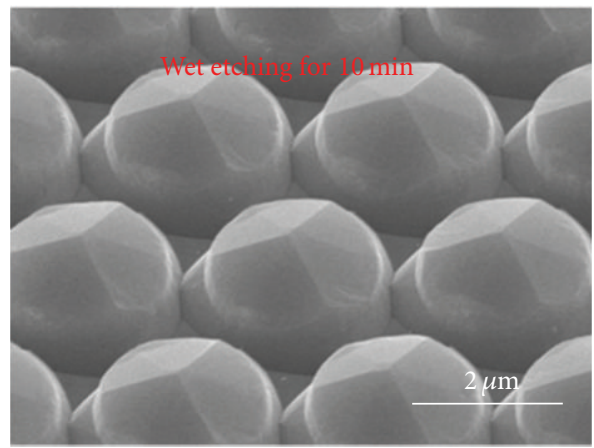

(e)

FIGURE 1: (a) Cross-sectional SEM image of cone-shaped PSS without treatment by wet etching and plane-view SEM images of cone-shaped PSSs subjected to wet etching for (b) $3 \mathrm{~min}$, (c) $5 \mathrm{~min}$, (d) $7 \mathrm{~min}$, and (e) $10 \mathrm{~min}$.

and the angular cone region at the initial stage, which results in an increased dislocation density and the deterioration of crystal quality in the epilayer.

Figures 3(a), 3(b), and 3(c) display the cross-sectional TEM images of the GaN epilayers grown on the PSSs subjected to wet etching for 0,3 , and 10 min, respectively. In comparison to Figures 3(a) and 3(c), an obvious difference can be observed in Figure 3(b) (i.e., the formations of protrusions and voids on the sidewalls of the cone pattern). Furthermore, we determined that no protrusion or void formed on the sidewalls of the cone pattern when the PSSs were wet-etched for $0,5,7$, and $10 \mathrm{~min}$ (the images of the GaN epilayers grown on the PSSs subjected to wet etching for 5 and $7 \mathrm{~min}$ are not shown here). This indicates that the special structure feature only appears in the GaN epilayer prepared on a PSS subjected to wet etching treatment for $3 \mathrm{~min}$. In addition, the right protrusion in Figure 3(b) was chosen to be examined using a selected area electron diffraction pattern, as shown in Figure 3(d). The single crystalline electron diffraction dots in Figure 3(d) indicate that the protrusion possesses the [1101] zone axis of GaN. Figures 4(a) and 4(b) show the cross-sectional SEM images before and after epilayer growth occurred on the PSS subjected to wet etching for $3 \mathrm{~min}$, respectively. We observed that the sidewalls of the cone patterns were smooth before wet etching, and the protrusions and voids were formed during epilayer growth. When we chose one of the cone patterns in Figure 4(b) (marked with a green circle) and enlarged the SEM magnification, the features of the protrusions and voids could be observed clearly, as shown in Figure 4(c). 


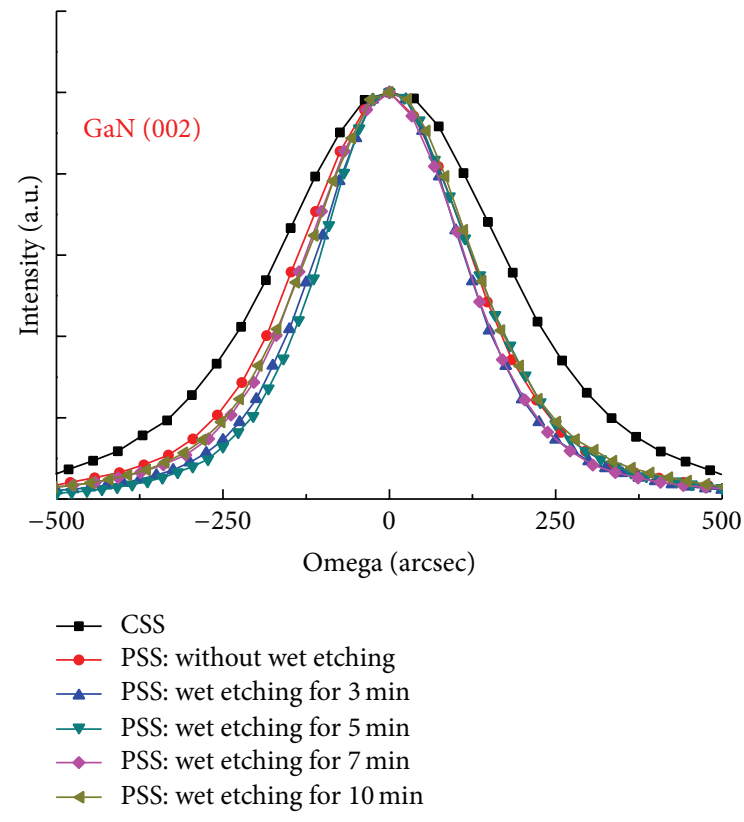

(a)

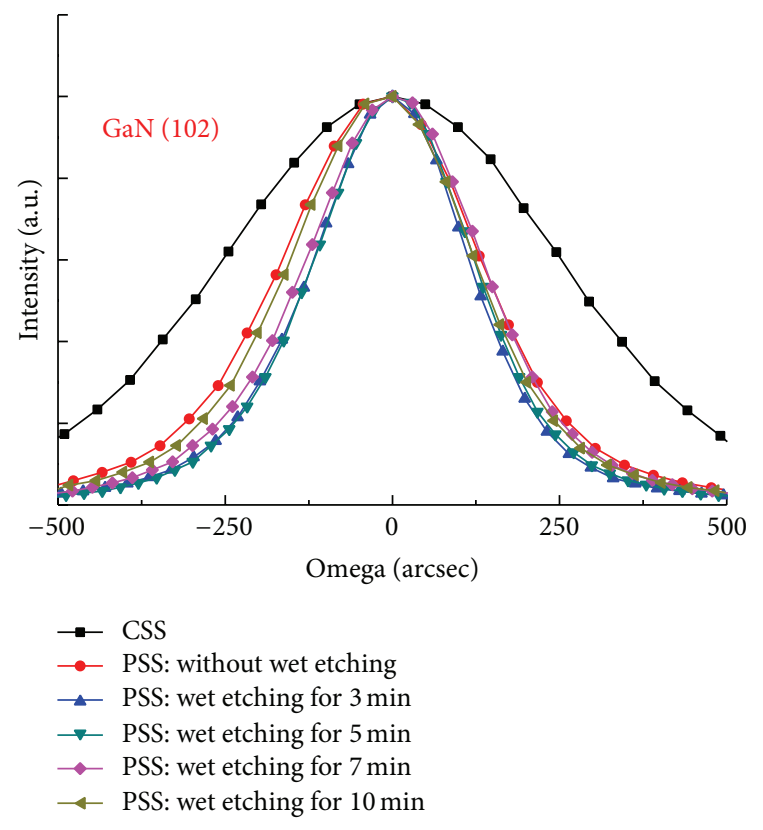

(b)

FIGURE 2: XRD FWHM values for the (a) (002) and (b) (102) reflections of GaN epilayers prepared on the CSS and various cone-shaped PSSs.

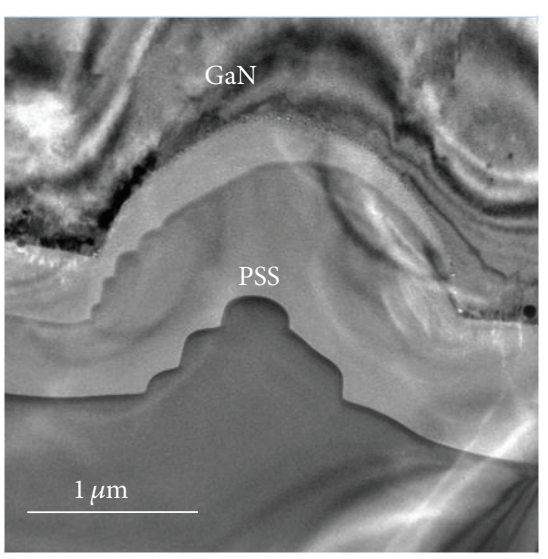

(a)

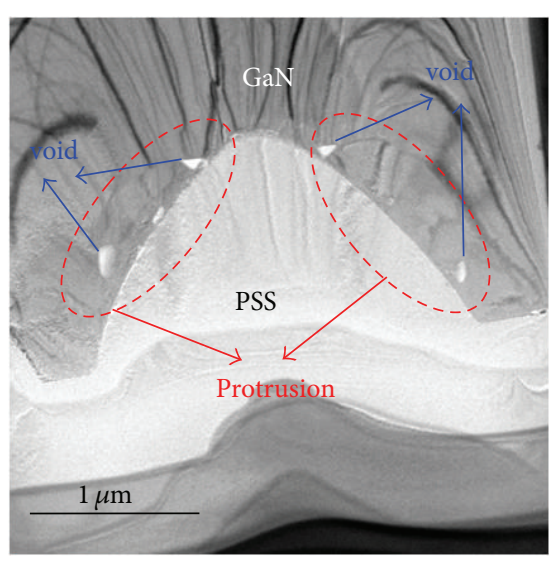

(b)

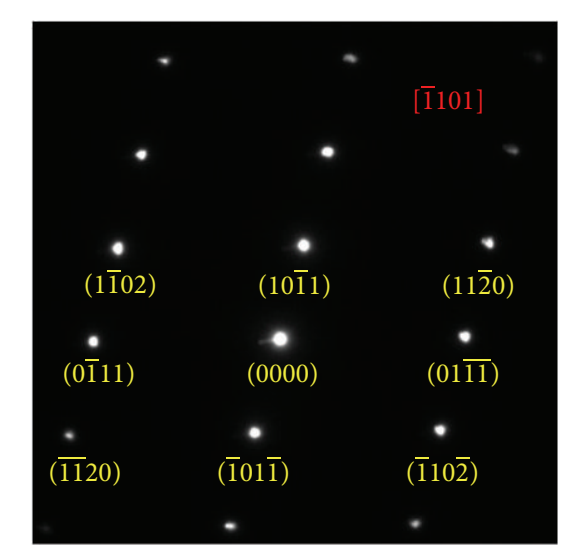

(d)

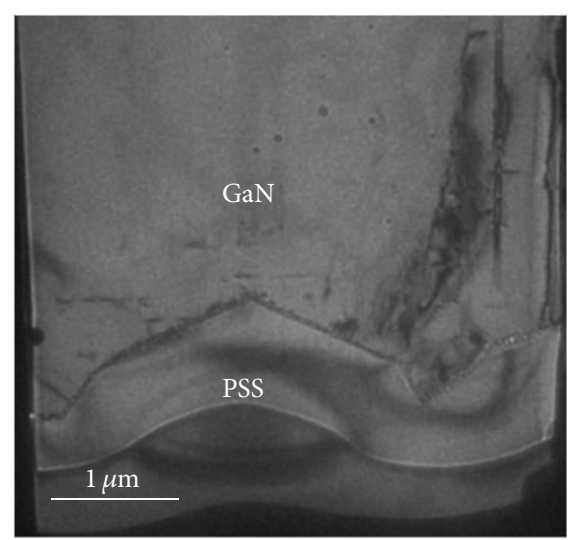

(c)

FIGURE 3: Cross-sectional TEM images of the GaN epilayers grown on the PSSs subjected to wet etching for (a) 0 min, (b) 3 min, and (c) $10 \mathrm{~min}$. (d) Selected area electron diffraction pattern of the right protrusion in (b). 


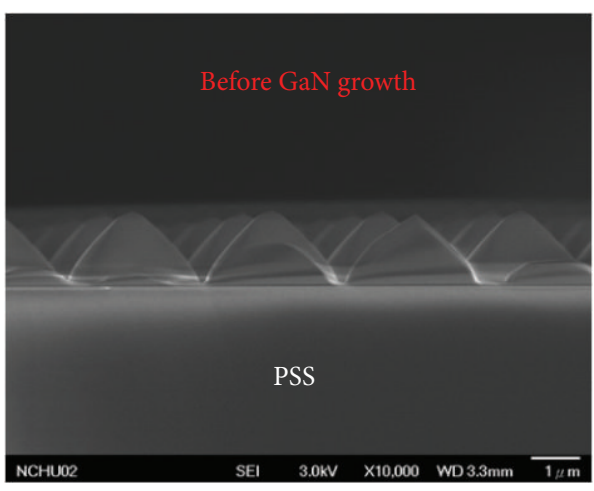

(a)

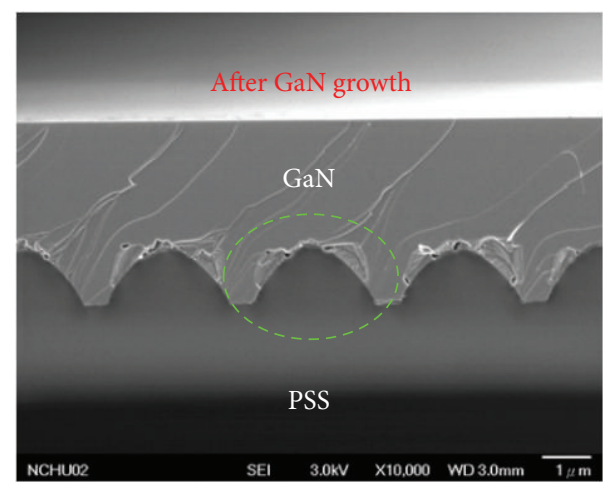

(b)

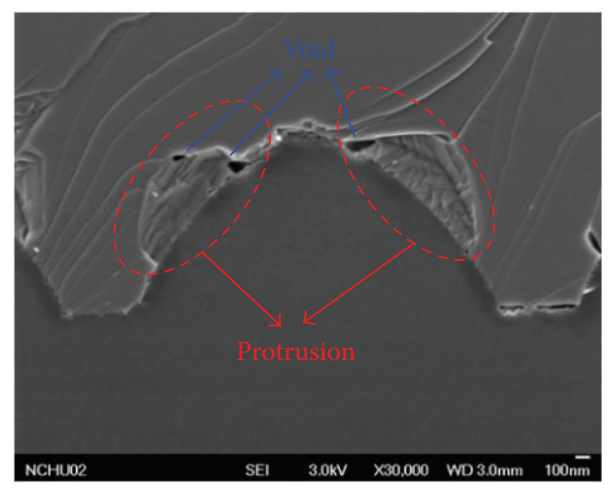

(c)

FIGURE 4: Cross-sectional SEM images (a) before and (b) after epilayer growth on PSS subjected to wet etching for 3 min and (c) SEM image taken at the cone pattern in (b) (marked with green circle) with higher magnification.

In this study, the formations of protrusions and voids were observed in the GaN growth on the cone-shaped PSS subjected to wet etching for $3 \mathrm{~min}$. The effects of the protrusions and voids on the epilayer quality and LED device performance are discussed. When the PSSs were employed, the GaN growth primarily occurred in the bottom regions (c-plane), and the ELOG process was used to merge the GaN films [9-11], leading to an improvement in the crystal quality of $\mathrm{GaN}$ epilayer. As the wet etching process was performed on the cone-shaped PSS for $3 \mathrm{~min}$, the indistinct angular planes formed on the sidewalls of the cone patterns. Subsequently, after growing the GaN epilayer on this PSS, the protrusions and voids were generated on the sidewalls of the cone patterns. Park et al. have proposed a detailed investigation of the microstructures of GaN epilayer grown on the coneshaped PSS [12]. It indicates that the recrystallized GaN islands and nanovoids were formed on the inclined surface of the cone-shaped PSS. Based on our observation, the features of the "recrystallized GaN islands and nanovoids" presented in Park et al. research are very similar to those of the "protrusions and voids" observed in our study. Additionally, the recrystallized GaN island was examined using a selected area electron diffraction pattern via the TEM measurement, and it revealed that the electron diffraction dots generated from the recrystallized GaN island possessed the [1101] zone axis of GaN. Consequently, we can confirm that the protrusions observed in our work are equivalent to the recrystallized GaN islands found in Park et al. research. According to Park et al. investigation, there is a $9^{\circ}$ rotation of the recrystallized $\mathrm{GaN}$ island to $\mathrm{GaN}$ epilayer and $\mathrm{Al}_{2} \mathrm{O}_{3}$ substrate, leading to further decrease in the lattice mismatch at the interfaces of $\mathrm{Al}_{2} \mathrm{O}_{3}$ /recrystallized $\mathrm{GaN}$ island and recrystallized $\mathrm{GaN}$ island/GaN epilayer. (Here, the GaN epilayer is meant for the GaN film with (002) growth orientation deposited on the recrystallized $\mathrm{GaN}$ island.) The $9^{\circ}$ rotation would result in a significant reduction of the misfit dislocation from the interface between the recrystallized $\mathrm{GaN}$ island and $\mathrm{GaN}$ epilayer. Furthermore, the crystal quality of GaN epilayer can be improved via the formation of recrystallized $\mathrm{GaN}$ islands. This implies that the protrusions observed in our study also play a key role in improving the quality of $\mathrm{GaN}$ epilayer and optoelectronic performance of LED. Besides, the enhancement in light extraction by embedding or forming voids in the LED structure has been reported in several studies [13-15]. Based on the aforementioned discussion, the formations of protrusions and voids in our work are indeed helpful for the improvements of epilayer quality and LED device performance. The device performance was discussed later, as shown in Figure 6. As discussed in Figure 2, with increasing the wet etching time on the PSS, the epilayer could be grown on both the bottom and the angular cone region at the initial stage, causing a degradation of the epilayer quality. 


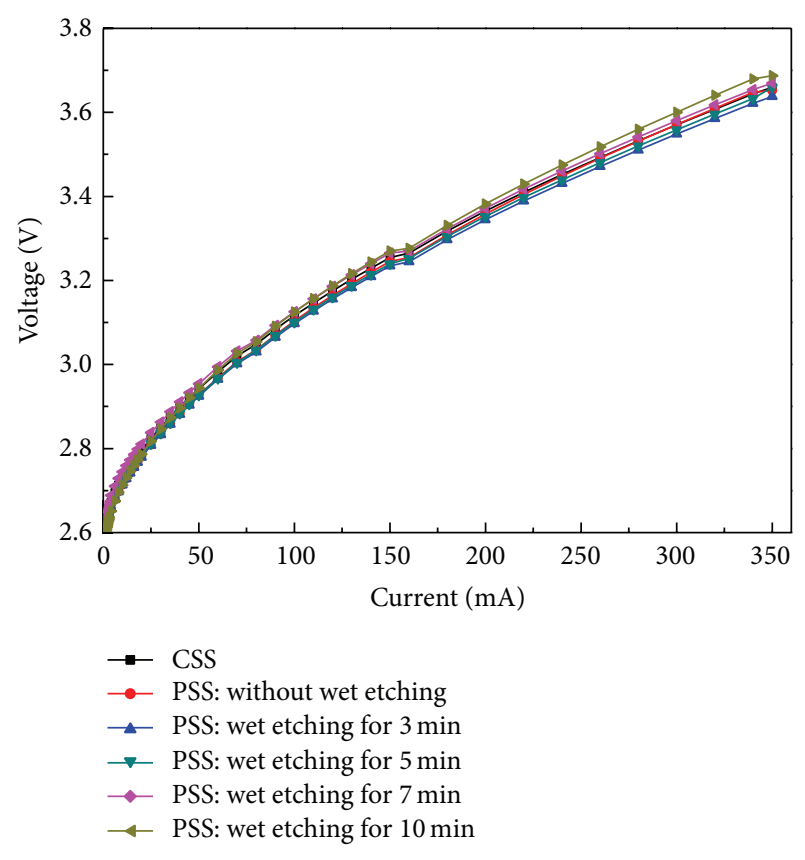

FIGURE 5: $I-V$ characteristics of the InGaN LEDs prepared on the CSS and various cone-shaped PSSs subjected to wet etching from 0 to $10 \mathrm{~min}$.

Moreover, the disappearance of $\mathrm{GaN}$ protrusions is the other reason for the degradation of the epilayer quality.

Figure 5 presents the $I-V$ characteristics of the LED samples fabricated on both the CSS and various cone-shaped PSSs. As the injection current increased to $350 \mathrm{~mA}$, the forward voltages of the LED samples prepared on the CSS and PSSs subjected to wet etching for $0,3,5,7$, and $10 \mathrm{~min}$ were determined to be $3.66,3.65,3.64,3.66,3.67$, and $3.69 \mathrm{~V}$, respectively. Figure 6 shows the light output power as a function of injection current ranging from 0 to $350 \mathrm{~mA}$ for the InGaN LEDs fabricated on both the CSS and various cone-shaped PSSs. As shown in Figure 2, the crystal quality of the GaN epilayer was enhanced efficiently by using the cone-shaped PSSs. A similar trend was observed in the device performance. Compared with the LED prepared on the CSS, the light output power of the LED grown on the cone-shaped PSS was substantially improved. Moreover, the light output power of the LED was further enhanced when the PSS was treated with wet etching because of the improvement in crystal quality of the GaN epilayer. Nevertheless, as the PSS was wet-etched for 5-10 min, the LED device exhibited a slight decrease in light output power. This was attributed to the deterioration of epilayer quality. At an injection current of $350 \mathrm{~mA}$, the light output power of the LED fabricated on a CSS was measured to be $237 \mathrm{~mW}$. In addition, the light output powers of the LEDs fabricated on PSSs subjected to wet etching for $0,3,5,7$, and 10 min were determined to be $333,367,356,353$, and $347 \mathrm{~mW}$, respectively. Compared with the LEDs prepared on the CSS and PSS without wet etching, the LED grown on the PSS subjected to wet etching for $3 \mathrm{~min}$ exhibited $55 \%$ and $10 \%$ improvements in light output power (at $350 \mathrm{~mA}$ ), respectively.

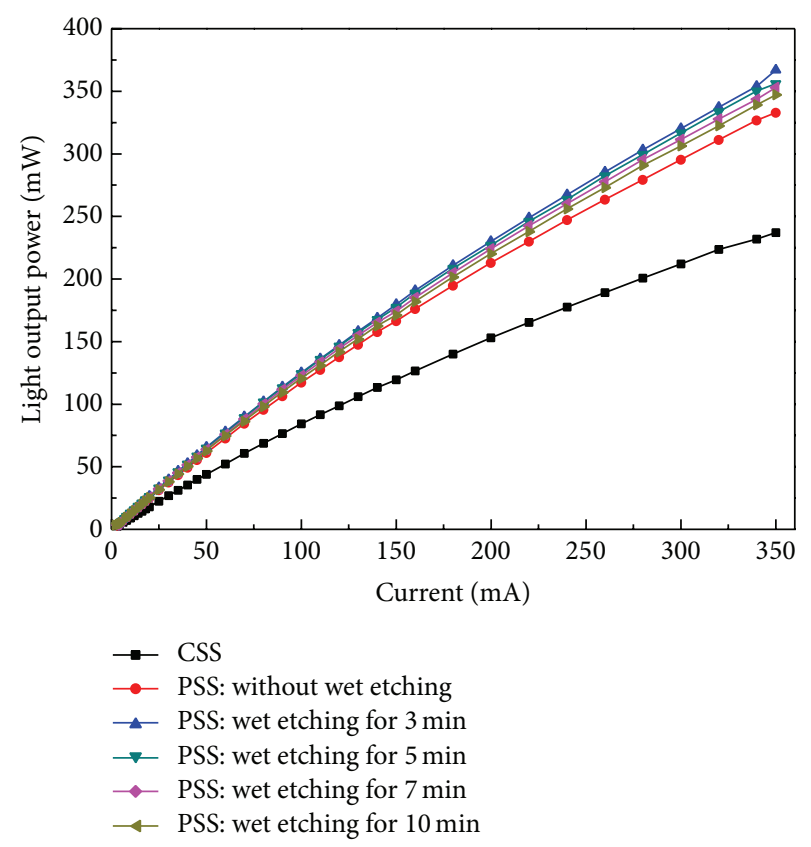

FIGURE 6: Light output power as a function of injection current of the InGaN LEDs prepared on the CSS and various cone-shaped PSSs subjected to wet etching from 0 to $10 \mathrm{~min}$.

\section{Conclusion}

In summary, InGaN LEDs were grown on the PSSs with modified top-tip cone shapes. The effects of various cone shapes on the epilayer quality and device performance were also investigated. Performing the wet etching treatment on the cone-shaped PSS resulted in the modification of the top tip of the pattern shape and the removal of dry-etched induced damage on the substrate surface. This led to the enhanced crystal quality of GaN growth and improved device performance. The optimal wet etching time for the coneshaped PSSs was determined to be $3 \mathrm{~min}$. The LED device prepared on this PSS demonstrated the most improvement in both epilayer quality and light extraction compared with those demonstrated by the other PSSs. This indicates that using the wet etching treatment on the cone-shaped PSSs is potentially useful in LED applications.

\section{Conflict of Interests}

The authors declare that there is no conflict of interests regarding the publication of this paper.

\section{Acknowledgments}

This work was supported by the Ministry of Economic Affairs under Grant no. 102-E0605 and National Science Council (Taiwan) under the Contract no. 102-2221-E-005-072-MY3. 


\section{References}

[1] S. Nakamura, T. Mukai, and M. Senoh, "Candela-class highbrightness InGaN/AlGaN double-heterostructure blue-lightemitting diodes," Applied Physics Letters, vol. 64, no. 13, pp. 1687-1689, 1994.

[2] H. X. Jiang, S. X. Jin, J. Li, J. Shakya, and J. Y. Lin, "III-nitride blue microdisplays," Applied Physics Letters, vol. 78, no. 9, pp. 1303-1305, 2001.

[3] C. Pan, C. Lee, J. Liu, G. Chen, and J. Chyi, "Luminescence efficiency of InGaN multiple-quantum-well ultraviolet lightemitting diodes," Applied Physics Letters, vol. 84, no. 25, pp. 5249-5251, 2004

[4] X. A. Cao, S. F. LeBoeuf, M. P. D'Evelyn et al., "Blue and near-ultraviolet light-emitting diodes on free-standing $\mathrm{GaN}$ substrates," Applied Physics Letters, vol. 84, no. 21, pp. 4313-4315, 2004.

[5] M. Iwaya, T. Takeuchi, S. Yamaguchi, C. Wetzel, H. Amano, and I. Akasaki, "Reduction of etch pit density in organometallic vapor phase epitaxy-grown $\mathrm{GaN}$ on sapphire by insertion of a low-temperature-deposited buffer layer between hightemperature-grown GaN," Japanese Journal of Applied Physics, vol. 37, no. 3B, pp. L316-L318, 1998.

[6] Y. B. Tao, T. J. Yu, Z. Y. Yang et al., "Evolution and control of dislocations in GaN grown on cone-patterned sapphire substrate by Metal Organic Vapor PhaseEpitaxy," Journal of Crystal Growth, vol. 315, no. 1, pp. 183-187, 2011.

[7] M. T. Wang, F. Brunner, K. Y. Liao, Y. L. Li, S. H. Tseng, and M. Weyers, "Optimization of GaN wafer bow grown on cone shaped patterned sapphire substrates," Journal of Crystal Growth, vol. 363, pp. 109-112, 2013.

[8] J. Lee, D. Lee, B. Oh, and J. Lee, "Comparison of InGaNbased LEDs grown on conventional sapphire and cone-shapepatterned sapphire substrate," IEEE Transactions on Electron Devices, vol. 57, no. 1, pp. 157-163, 2010.

[9] J. Lee, J. T. Oh, Y. C. Kim, and J. Lee, "Stress reduction and enhanced extraction efficiency of GaN-based LED grown on cone-shape-patterned sapphire," IEEE Photonics Technology Letters, vol. 20, no. 18, pp. 1563-1565, 2008.

[10] K. Hiramatsu, K. Nishiyama, M. Onishi et al., "Fabrication and characterization of low defect density GaN using facetcontrolled epitaxial lateral overgrowth (FACELO)," Journal of Crystal Growth, vol. 221, no. 1-4, pp. 316-326, 2000.

[11] H. Lahrèche, P. Vennéguès, B. Beaumont, and P. Gibart, "Growth of high-quality GaN by low-pressure metal-organic vapour phase epitaxy (LP-MOVPE) from 3D islands and lateral overgrowth," Journal of Crystal Growth, vol. 205, no. 3, pp. 245252, 1999.

[12] J. S. Park, J. M. Yang, K. J. Park et al., “Transmission electron microscopy study of microstructural properties and dislocation characterization in the GaN film grown on the cone-shaped patterned $\mathrm{Al}_{2} \mathrm{O}_{3}$ substrate," Microscopy, vol. 63, no. 1, pp. 15-22, 2014.

[13] C. Cho, M. Kwon, I. Park et al., "High-efficiency light-emitting diode with air voids embedded in lateral epitaxially overgrown GaN using a metal mask," Optics Express, vol. 19, no. 4, pp. A943-A948, 2011.

[14] C. H. Chiu, C. C. Lin, H. V. Han et al., "High efficiency $\mathrm{GaN}$-based light-emitting diodes with embedded air voids $/ \mathrm{SiO}_{2}$ nanomasks," Nanotechnology, vol. 23, no. 4, Article ID 045303, 2012.
[15] S. M. Kim, K. H. Lee, and G. Y. Jung, "Epitaxial lateral overgrowth on the air void embedded $\mathrm{SiO}_{2}$ mask for InGaN light-emitting diodes," CrystEngComm, vol. 15, no. 31, pp. 60626065, 2013. 

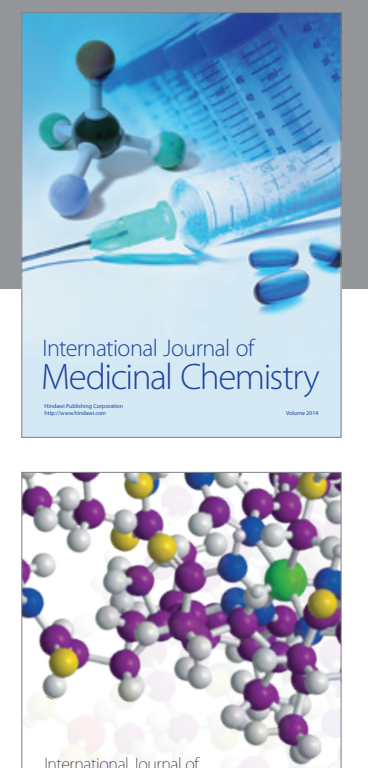

\section{Carbohydrate} Chemistry

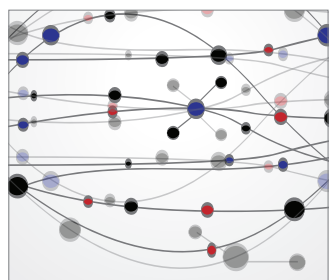

The Scientific World Journal
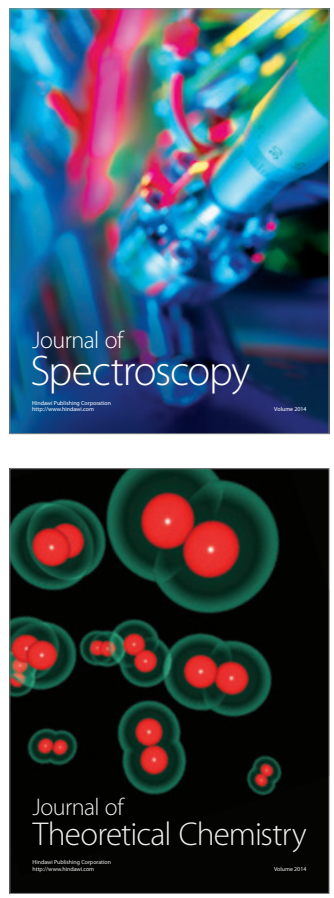
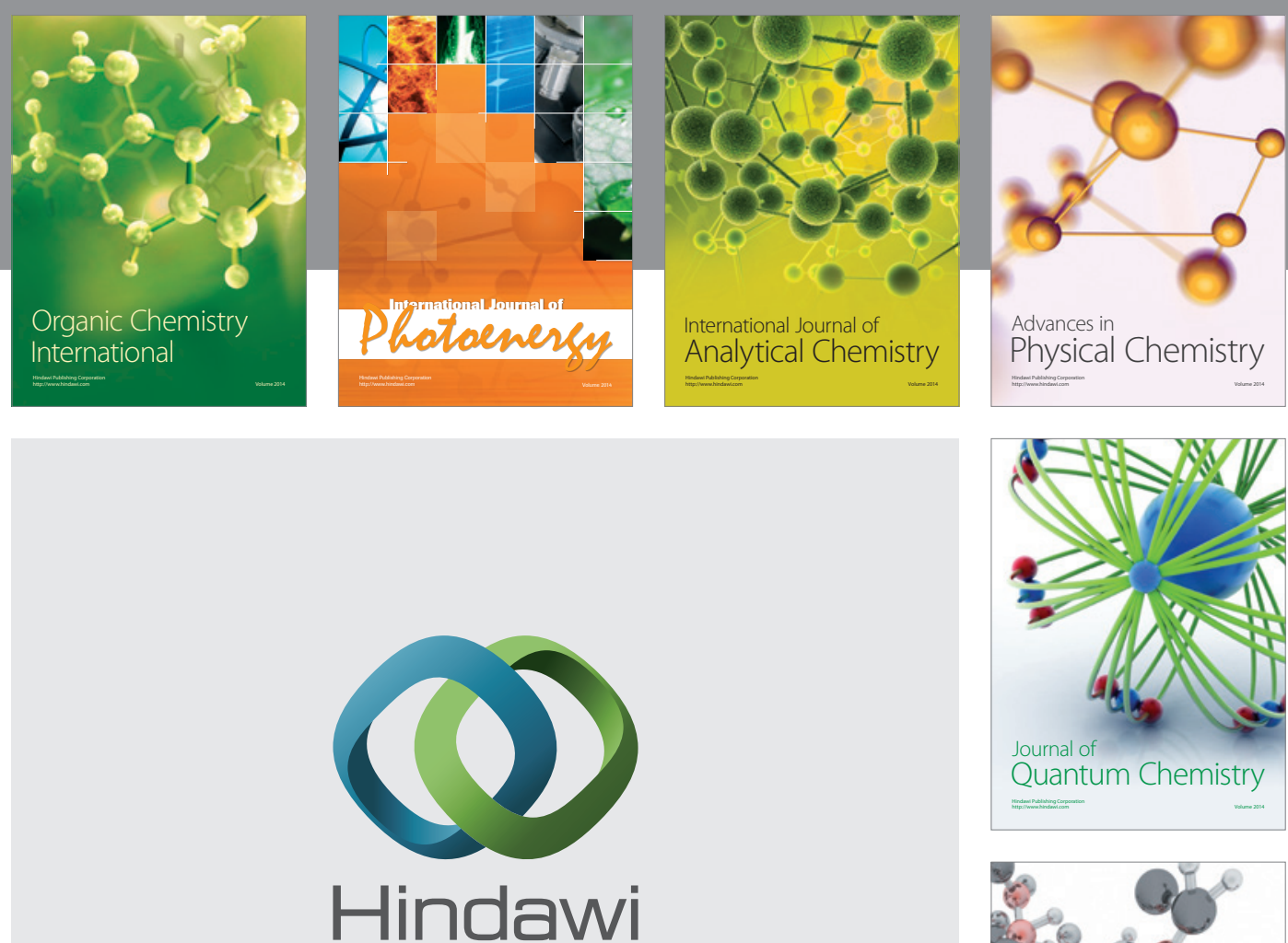

Submit your manuscripts at

http://www.hindawi.com

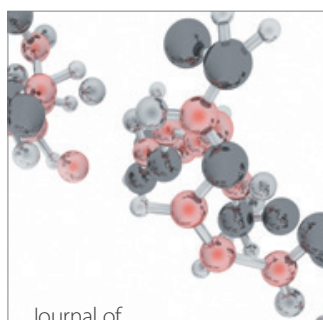

Analytical Methods

in Chemistry

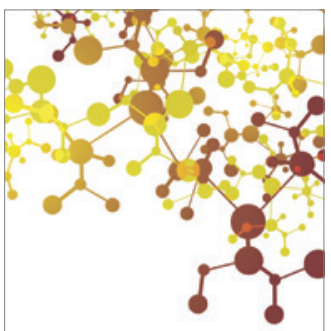

Journal of

Applied Chemistry

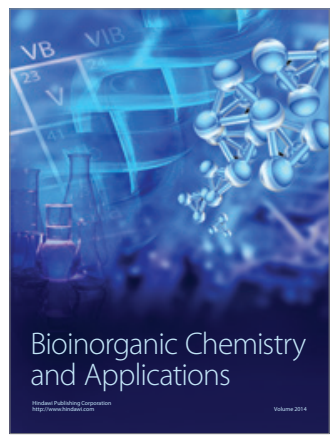

Inorganic Chemistry
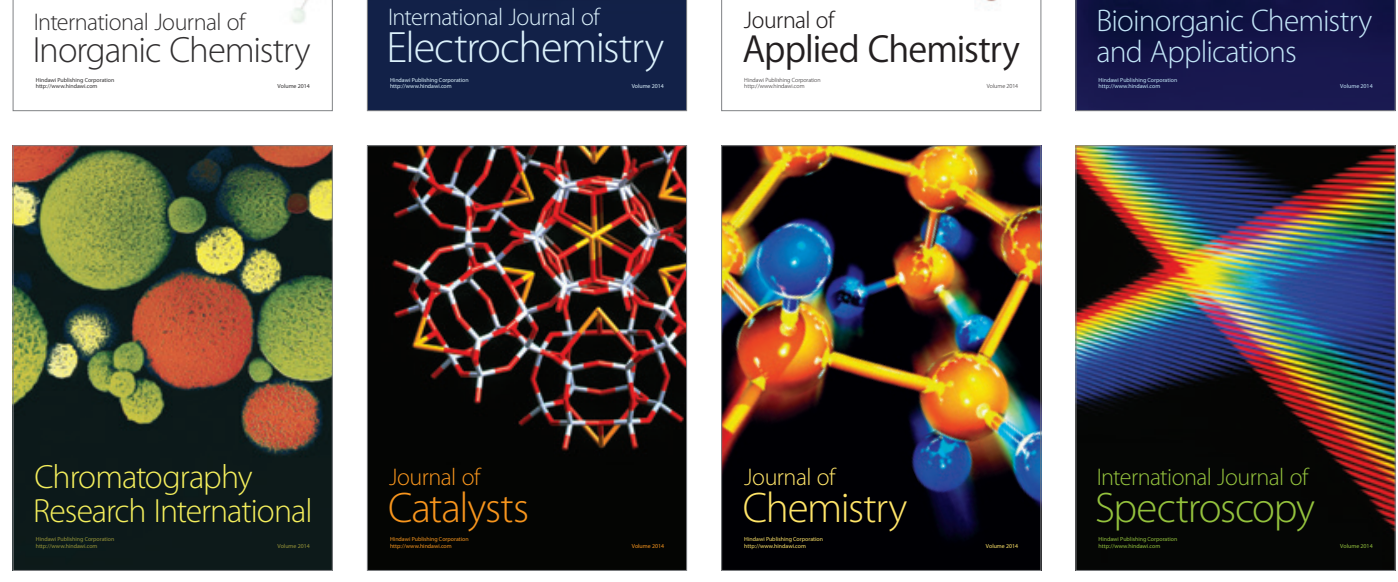